\title{
Microsurgical open mini uniskin incision technique in the surgical treatment of carpal tunnel syndrome
}

\author{
Aydin Keramettin, Cokluk Cengiz, Cengiz Nilgun, Bilgici Ayhan \\ Department of Neurosurgery, Medical Faculty, Ondokuzmayis University, Samsun, Turkey
}

Background: Patients who undergo carpal tunnel surgery sometimes complain of the restriction of the grip and pinch function, palmar tenderness, cosmetic problems, and scar formation at the site of the incision. Aims: We used a modified mini uni-skin incision with appropriate hand position for microscopic view in the surgical treatment of carpal tunnel syndrome to prevent cosmetic problems related with scar formation after surgery. Settings and Design: In this study we used two different skin incision techniques; mini uni-skin incision and standard incision. In mini uni-skin incision technique the hands were positioned in a way that the wrist are hyperextended. A small skin incision one $\mathrm{cm}$ long was done from the inferior flexion crease towards the point between the thirth and fourth fingers. Materials and Methods: Standard incision and mini uni-skin incision were compared according to their cosmetic result, grip and pinch function, palmar tenderness, and painful scar formation. Statistical analysis: Student $t$ test was used for this study. Results: $56(43 \%)$ patients were operated with mini uni-skin incision, and $73(57 \%)$ cases were operated with standard incision. The scores of grip, pinch and cosmetic results were better in the patients who were operated with mini uni-skin incision technique from those of standard incision. Conclusion: In this clinical study we used a modified skin incision (mini uni-skin incision) technique in the surgical treatment of carpal tunnel surgery. Our results revealed that mini uni-skin incision is superior from the standard incision.

Key words: Mini open uniskin incision, open median nerve release, carpal tunnel syndrome.

\section{Introduction}

Carpal tunnel syndrome (CTS) is the most common entrapment neuropathy, accounting for more than 200,000 surgical procedures per year in the United States. Approximately $1 \%$ of population is affected by carpal tunnel syndrome. ${ }^{[1,2]}$ Common clinical presentations include painful paresthesias or burning pain in the lateral half of the hand and the radial three fingers. ${ }^{[1]}$

History, physical examination, electromyography, and nerve conduction velocity measurements are essential for the diagnosis. ${ }^{[3]}$ Open carpal tunnel release has been widely used as a surgical alternative for patients who are resistant to the conservative treatments. However, although its effectiveness has been recognized, open carpal tunnel release has been associated with problems such as hypertrophic scarring, scar tenderness and delayed rehabilitation. ${ }^{[4,5]}$ Various limited skin incisions and endoscopic techniques have been proposed as a minimally invasive and effective for preventing of the excessive sear formation and the achieving of a better cosmetic results. ${ }^{[1,3,6-9]}$

In this clinical study, we described a modified surgical technique (mini-open uni-skin incision), and compared it with the standard incision according to the capability of grip and pinch function, cosmetic results, palmar tenderness and painful sear formation.

\section{Materials and Methods}

Local ethical committee (Ondokuzmayis University, Medical Faculty, Local Ethical Committee) approved this clinical study. Before operation all patients and/or their family were informed about the type of the operation.

This retrospective study included 129 female right hands with carpal tunnel syndrome underwent microsurgical intervention for median nerve release using mini-uniskin incision and standard incision between 2001 and 2005. The diagnosis was made from the patient's history, physical examination, the results of electromyographic studies, and nerve conduction velocities in all cases. Patients with left and bilateral carpal tunnel syndrome were not included to the study. Follow up examinations of the cases were made 20, 60, and 90 days after the operation. A dermatologist and a doctor of physical medicine examined all patients in the aspect of cosmetic outcome, grip and pinch functions. The patients 
were examined in the aspect of grip and pinch function, palmar tenderness, and painful scar formation.

We used some simple questions selected from the daily activities in the evaluation of grip and pinch functions. Table 1 showed the questions for evaluating of these function. Grip and pinch function, palmar tenderness, and scar formation were clinically graded four level as poor, moderate, good, and excellent in according to patient's description and physical examination. Numerical values were given for each levels, 0 for poor, 1 point for moderate, 2 point for good, and 3 point for excellent. Student- $t$ test was used for statistical analysis.

\section{Surgical technique}

All patients were operated in supine position. The affected hand was positioned on the hand holding apparatus of the operating table. Before surgery, the forearm, wrist, and hand were cleaned with povidone iodine solution (Isosol $1000 \mathrm{ml}$; Merkez laboratuari, Istanbul, Turkey) for providing a sterile surgical condition. The line of surgical incision was drawn with a sterile surgical pen. The surrounding area was covered with sterile cotton compress to isolate the hand. The operation was performed under local anesthesia induced with $2 \%$ prilocain hidrocloride (Citanest 20 ml; Flacon Astra Zeneka, Istanbul, Turkey) No tourniquet was used in any of the operation. An operative microscope (Carl Zeiss contraverse microscope, Opmi CS-NC) was used in all operations.

\section{Standart incision technique}

The details of standard skin incision for carpal tunnel surgery were previously described in the published medical literature. ${ }^{[10]}$ Rengachary previously detailed the standard incision used in this study. ${ }^{[10]}$ He described that the standard skin incision for carpal tunnel surgery is made from the wrist flexion crease and extends curvilinearly to a point in line with the distal border of the fully extended thumb. In this technique, the incision is generally placed 2 to $3 \mathrm{~mm}$ medial to the volar crease, in line with the long axis of the ring finger.

\section{Mini uni-skin incision technique}

The hands were extended as much as possible in physiologic bordes. Operative microscope is mandatory for this technique. The view of a surgeon from the operating microscope should be along the carpal tunnel. Perpendicularly, the palmar plane of the hyper extended hand and view plane of the operating microscope

\section{Table 1: Questions for evaluating of grip and pinch} functions

\section{Gripping functions}

1. Gripping of the stick of an electrical vacuum

2. Gripping of the handle of a knife

3. Gripping of the handle of a door

4. Gripping of the stalk of a hair-drying machine

\section{Pinching functions}

1. Pinching of a key, turning it to open a door

2. Pinching of a pen and writing

3. Pinching of a toothbrush and brushing of the teeth

4. Pinching of a screwdriver should be in same plane [Figure 1). The incision was located at the superior region of the palm. There is a triangle on this area. Distal flexion crease is the base of this triangle. The thenar crease created lateral edge of this triangle. The hypo-thenar crease is the medial border of this triangle. The tip of this triangle continues with the inter-thenar crease. The incision was started from just distal to the wrist flexion crease on midline of the triangle and extended for $1 \mathrm{~cm}$ to the tip of the triangle [Figure $2 \mathrm{~A}$ ).

A mini skin retractor was used to retract the incisioned skin [Figure 2B). The subcutaneous fat tissue was dissected laterally. 15 no surgical blade was used to open proximal part of the ligament. After this maneuver, the ligament was cut with a micro scissors. Our experience showed us that the maneuver of the hyperextension of the hand provide a good surgical route with the depletion of the median nerve and the tendons of the fingers to the posterior bony wall of the tunnel. Because of this, the sectioning of ligament with scissor is safe and reliable.

The surgical area was then irrigated with sterile saline solution and homeostasis was obtained with bipolar coagulation. The sectioning of the ligament was checked along the carpal tunnel, and the median nerve was inspected [Figure 2C). The skin was sutured with interrupted 4-0 nylon sutures without subcutaneous suture. The mean operating time was 30-45 minutes. The skin sutures were removed 10 days after the operation.

\section{Results}

The present study includes 129 female wrists with carpal tunnel syndrome aged between 36-72 years old. All patients had the evidence of median nerve entrapment at the wrist in the

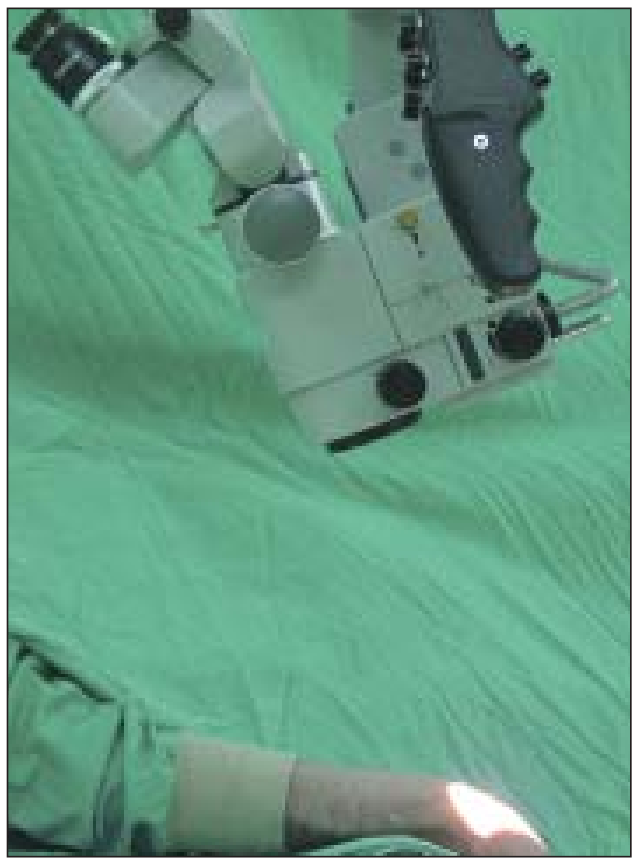

Figure 1: The position of the operating microscope was shown in this figure. The direction of the operating microscope should be positioned in perpendicular plane with flexor retinaculum 


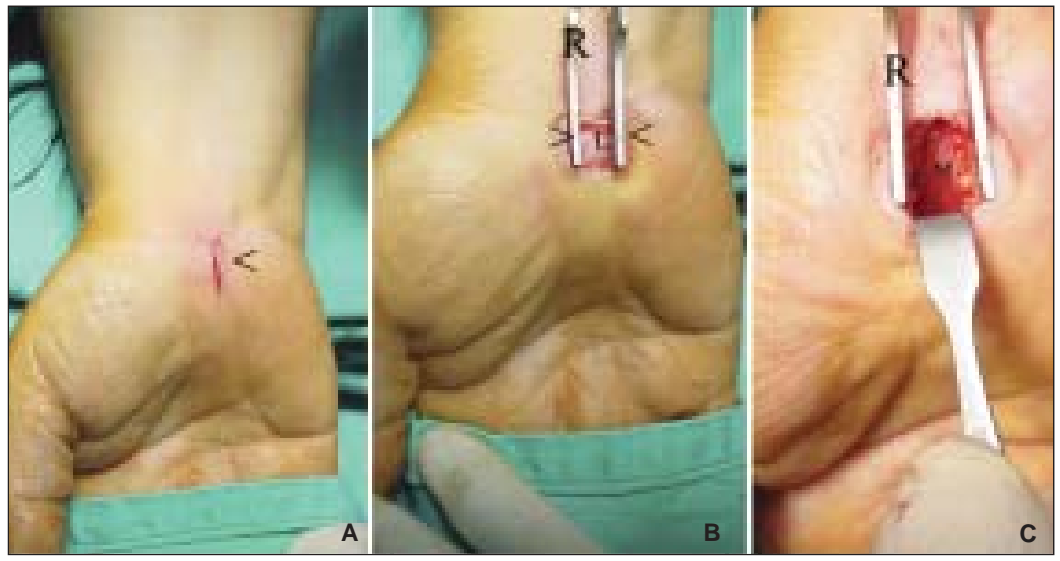

Figure 2: A. The skin incision about $1 \mathrm{~cm}$ in length at just distal to the distal flexion crease (arrow shows the skin incision). B. The appearance of the transverse carpal ligament after retraction of the skin with small self-retaining retractor (R: self-retaining retractor, L: transverse carpal ligament). C. The appearance of median nerve after sectioning of the transverse carpal ligament (M: median nerve, R: self retaining retractor).

electromyographic studies and nerve conduction velocities. 56 $(43 \%)$ of them were operated with mini uni-skin incision, and remaining 73 (57\%) were operated with standard incision.

The mean follow up period was 2 years. There was no reoperation, skin infection, and palmar cutaneous branch injury in all groups. Table 2 shows the scores of grip and pinch function, palmar tenderness, cosmetic results, and postoperative course for patients operated with both methods. The scores of grip, pinch and cosmetic results were better in the patients who were operated on with mini uni-skin incision technique from those of standard incision in the ratio of $26 \%, 17 \%$, and $54 \%$ respectively. Palmar tenderness was lower in patients operated on with mini uni-skin from those of standard incision. The differences between these two groups are statistically significant $(P<0.01)$.

\section{Discussion}

If a surgery is necessary for median nerve decompression, the surgical technique should be less traumatic and minimally invasive for both of median nerve and palmar skin to prevent the postoperative sequels and achieve a good postoperative course without persistent symptoms. Damage of the palmar cutaneous branch of the median nerve, reflex sympathetic dystrophy, hypertrophic scar formation, scar sensitivity, palmar hematoma, bowstringing of the flexor tendons, double crush, and adherence of the flexor tendons had been reported as the causes of persistent symptoms after median nerve release. ${ }^{[11-14]}$ The most common and less serious sequels of carpal tunnel surgery related with skin incision are hypertrophic scar formation, sensitive scar, tenderness and cosmetic dissatisfaction. ${ }^{[1,2]}$ The incidence of these types of sequels has been reported in some series at $24 \%$. ${ }^{[1]}$

Standard open procedures with different type skin incisions; endoscopic techniques have been described for surgical division of the transverse carpal ligament. ${ }^{[3,6,-9,11,15-19]}$

The aim of this clinical study was to use a shorter skin incision to reduce the number of sequelae of the carpal tunnel surgery related with skin incision and compare this with the results of the standard skin incision technique. Open mini uni-skin incision is different from standard technique in terms of localisation and length of the incision, and position of the hand under the operative microscope. We identified a triangle on the proximal part of the palm in the localization of the skin incision. The skin is relatively thin, and biomechanically less movable in this triangle in comparison with other parts of the palm. This incision also facilitates the release of proximal transverse carpal ligament.

We standardized our patient population by selection of righthanded women. Questions for evaluating of the grip and pinch function includes some functions commonly used by women in daily activities. The patients described the capability of their hands during making of these activities such as poor, moderate, good, and excellent. The mean scores of grip function were found better in the ratio of $26 \%$ in the patients who were operated with mini uni-skin incision technique. The scores of pinch function were also better in the ratio of $17 \%$ in the patients operated with mini uni-skin incision.

The clinical data from the present series suggested that cosmetic

Table 2: The scores of grip, pinch, palmar tenderness, cosmetic results and postoperative courses of the patients operated with mini uni-skin incision and standard incision

\begin{tabular}{lccccc}
\hline Type of incision & Grip & Pinch & $\begin{array}{c}\text { Palmar } \\
\text { tenderness }\end{array}$ & $\begin{array}{c}\text { Scar tissue and } \\
\text { cosmetic results }\end{array}$ & $\begin{array}{c}\text { Postoperative } \\
\text { course }\end{array}$ \\
\hline Mini uni-skin incision & $1.79 \pm 0.62$ & $1.89 \pm 0.78$ & $2.79 \pm 0.42$ & $2.42 \pm 0.87$ & $2.56 \pm 0.5$ \\
Standard incision & $1.31 \pm 0.47$ & $1.56 \pm 0.51$ & $1.12 \pm 0.61$ & $1.12 \pm 0.61$ & $1.87 \pm 0.5$ \\
& $t=4.8$ & $t=2.7$ & $t=18.4$ & $t=9.5$ & $t=7.76$ \\
& $\mathrm{SD}=127$ & $\mathrm{SD}=127$ & $\mathrm{SD}=127$ & $\mathrm{SD}=127$ & $\mathrm{SD}=127$ \\
& $P<0.01$ & $P<0.01$ & $P<0.001$ & $P<0.01$ & $P<0.001$ \\
\hline
\end{tabular}


results of mini uni-skin incision were superior from those of standard skin incision. Sensitive scar formation and tenderness were also less in mini uni-skin incision group from those of standard incision. The differences between these two groups were statistically significant.

Although in large series, cosmetic problems originated from excessive scar formation seems relatively uncommon problem after carpal tunnel surgery. But it is clear that palmar skin surface lost their biomechanical movements and elasticity after surgery even in normal wound healing process. The limited skin incision occupies small area on the palmar surface of the hand. This provides more movement capability, elasticity and better appearance to the skin surface.

\section{Conclusion}

The mini uni-skin incision technique for median nerve release can be used in the surgical treatment of carpal tunnel syndrome to achieve a better palmar appearance. Our clinical data revealed that this technique is superior to standard incision in the aspect of grip and pinch function, and cosmetic results. Our observation revealed that the patients operated with mini uni-skin incision technique have an easier postoperative course in comparison with standard incision.

\section{References}

1. Huang JH, Zager EL. Mini open carpal tunnel decompression. Neurosurg 2004; 54(2): 397-9.

2. Hudson AR, Wissinger JP, Salazar JL, Kline DG, Yarzagaray L, Danoff D, Fernandez E, Field EM, Gainsburg DB, Fabi RA, Mackinnon SE. Carpal tunnel svndrome. Surg Neurol 1997;47:105-14.
3. Cokluk C, Senel A, Iyigun O, Celik F, Rakunt C. Open median nevre release using double mini skin incision in patients with carpal tunnel syndrome:technique and clinical results. Neurol Med Chir (Tokyo). 2003;43:465-7.

4. Louis DS, Greene TL, Noellert RC. Complications of carpal tunnel surgery. .J Neurosurg. 1985; 62: 352-6.

5. Okada M, Tsubata O, Yasumoto S, Toda N, Matsumoto T. Clinical study of surgical treatment of carpal tunnel syndrome. Open versus endoscopic technique. Journal of Orthopaedic Surgery. 2000;8:19-25.

6. Muller LP, Rudig L, Degreif .J, Rommens PM. Endoscopic carpal tunnel release: results with special consideration to possible complications. Knee Surg Sports Traumatol Arthrose. 2000;8:166-72.,

7. Nathan PA. Carpal tunnel release using minimally invasive technique. Plast Reconstr Surg. 1997;99:1195-6.

8. Lee WP, Strickland JW. Safe carpal tunnel release via a limited palmar incision. Plast Reconstr Surg 1998;101:418-24.

9. Agee JM, McCarroll HR Jr, Tortosa RD, Berry DA, Szabo RM, Peimer CA Endoscopic release of the carpal tunnel: a randomized prospective multicenter study. J Hand Surg [Am]. 1992; 17: 987-95.

10. Rengachary S. Entrapment neuropathies, In Wilkins R, Rengachary S (eds): Neurosurgerv. New York, McGraw-Hill Book co.,pp 1771-76.

11. Chow JC. Endoscopic release of the carpal ligament. A new technique for carpal tunnel syndrome. Arthroscopy 1989; 5: 19-24.

12. Mirza MA, King ET Jr. Newer techniques of carpal tunnel release. Orthop Clin North Am. 1996; 27: 355-71.

13. Louis DS, Greene TL, Noellert RC. Complications of carpal tunnel surgery. .J Neurosurg. 1985; 62: 352-6.

14. Shapiro S. Microsurgical carpal tunnel release. Technique and application. Neurosurg. 1995; 37: 66-70.

15. Bradley MP, Hayes EP, Weiss AP, Akelman E. A prospective study of outcome following mini-open carpal tunnel release. Hand Surg (Singapore) 2003; 8: 59-63.

16. Kiymaz N, Cirak B, Tuncay I, Demir O. Comparing open surgery with endoscopic releasing in the treatment of carpal tunnel syndrome. Minim Invasive Neurosurg 2002; 45: 228-30

17. Jugovac I, Burgic N, Micovic V, Radolovic-Prenc L, Uravic M, Golubovic V, Stancic MF. Carpal tunnel release by limited palmar incision vs traditional open technique: randomized controlled trial. Croat Med J (Croatia) 2002; 43: 33-6.

18. Klein RD, Kotsis SV, Chung KC. Open carpal tunnel release using a 1-centimeter incision: technique and outcomes for 104 patients. Plast Reconstr Surg. 2003; 111: $1616-22$

19. Ahcan U, Arnez ZM, Bajrovic F, Zorman P. Surgical technique to reduce sear discomfort after carpal tunnel surgerv.J Hand Surg [Am]. 2002;27: 821-7. 